\title{
Systematic Review and Meta-Analysis of Published Literatures: How Much do We Know about Reliable Prevalence of Leptospirosis in Iran? \\ H Khayat $^{1}$, K Sayehmiri ${ }^{2}$, F Sayehmiri ${ }^{1}$, S Ghafourian $^{1}$, M Rezapour ${ }^{3}$, A Sheikhian ${ }^{4}$, L Bogdanovic ${ }^{5}$, M Taherikalani ${ }^{3,8}$
}

\begin{abstract}
Deficiency in knowledge about leptospirosis prevalence in Iran led us to clarify the prevalence of Leptospirosis and its diagnostic tests and increase global awareness of the diseases in this era via systematic review and Meta-analysis. A comprehensive search about the prevalence of Leptospirosis was done in PubMed, ISI Web of Sciences, Cochran Library, EMBAS, Scopus, Magiran, Iranmedex, Scientific Information Database (SID), and Google scholar from 1995 to 2013. Twenty two relevant papers were found and analyzed using R software and comprehensive meta-analysis software Biostat V2.0.

The meta-analysis findings demonstrated that the prevalence of Leptospirosis in Iran was 39\% (95\% Confidence Interval). Our analysis showed that the highest prevalence was observed in the province of Mazandaran (44\%) (95\% CI 27-61). While, interestingly, the lowest prevalence occurred in its neighboring province (Golestan, 34\%) (95\% CI 15-83). Further stratified analysis highlighted that farmers were a population at risk (65\% (95\% CI 74-57)) and that the disease is more prevalent in males (69\% (95\% CI 74-56). Based on the information in the current study, we induced that Leptospirosis occurred highly in humid climate areas of northern Iran. Due to the lack of Leptospirosis specific clinical symptoms and differences in sensitivity and specificity of diagnostic tests, we recommend doing further comprehensive studies by reliable tests and establishing national and local controlling programs for public awareness and reduction of human exposure to infected animals and their tissue and fluids.
\end{abstract}

Keywords: Iran leptospirosis, meta-analysis

From: ${ }^{1}$ Clinical Microbiology Research Centre, Ilam University of Medical Sciences, Ilam, Iran, ${ }^{2}$ Department of Epidemiology, School of Medicine, Ilam University of Medical Sciences, Ilam Iran, ${ }^{3}$ Razi Herbal Medicines Research Centre, Lorestan University of Medical Sciences,

${ }^{4}$ Department of Immunology, School of Medicine, Lorestan University of Medical Sciences, Khorramabad, Iran, and ${ }^{5}$ Department of Public Health, University of Naples Federicl II Naples, Italy.

Correspondence: Dr M Taherikalani, Razi Herbal Medicines Research Centre and Department of Microbiology School of Medicine 1Orestan University of Medical Sciences, Khorramabad, Iran. Fax: +98 66-33227593, e-mail: taherikalani@gmail.com 


\section{INTRODUCTION}

Leptospirosis is known as the most common zoonosis in the world that is caused by spirochetes

belonging to the genus Leptospira (1). It is also settled in tropical and subtropical regions (2). Transmission route of Leptospira spp to human is debated in different countries $(3,4)$. The majority of researches demonstrated direct or indirect exposure to animals such as rodents and livestock and also poor sanitation, urban overcrowding, poor waste disposal, heavy rainfall and floods are the main risk factors involved in the development of Leptospirosis $(3,4)$. Although, clinical profiles of Leptospirosis have changed in recent years but most of the human infections manifest a wide spectrum of disease from mild flu like and self-limited illness to fulminant multi-system involvement with acute renal failure and hemorrhage leading rapidly to death (5-8). Unfortunately, there is a lack of reliable knowledge on the prevalence of Leptospirosis in Iran, due to mild and self-limited clinical manifestation and/or lack of awareness of physicians about the diseases. So, the current review is an attempt to determine the prevalence of Leptospirosis in Iran based on a comprehensive systematic review and meta-analysis of published literatures.

\section{METHODS}

\section{Data Source}

The data about the epidemiology of Leptospirosis in Iran as well as period, location, sample size, diagnostic methods, gender, occupation, and age groups were obtained from reputable published articles during the years 1995 to 2012 (table 1). Most of the studies were conducted in Gilan $(\mathrm{n}=10 ; 45.4 \%)$ and Mazandaran $(\mathrm{n}=5 ; 22.7 \%)$ and the majority of diagnostic methods for the 
detection of Leptospirosis in these studies were MAT $(n=10 ; 45.4 \%)$ and ELISA $(n=8 ; 36.4 \%)$. The search terms were Leptospirosis; Leptospira, Weil's disease, and Iran. International main databases including ISI web of Knowledge, Medline/PubMed, Scopus, EMBASE and Cochrane Library which were searched by two independent researchers. In order to increase the number of results, Google Scholar and current Persian databases (IranMedex, IranDoc, SID and Magiran) were also searched. The Persian keywords were equivalent to the English keywords and all of the probable combinations were also considered.

\section{Study Selection and Data Extraction}

All results were screened and those that were not relevant to the review and/or duplicates were removed (Flowchart 1). All desired information such as study period, location, sample size, diagnostic methods, gender, occupation, and age groups were entered into data collection forms, and then transferred into Microsoft Excel.

\section{Statistical Analysis}

According to the main objective of Leptospirosis prevalence in Iran, we estimated variance by binominal distributions. Prevalence and 95\% confidence interval was calculated using random effect model for meta-analysis. To pool prevalence reported by different studies, weighting averaging was used. Each study given a weight equal to its inverse variance. Q test and I2 index, at the type I error of smaller than 0.10 , were applied in order to evaluate heterogeneity. Whenever the results of the study were heterogeneous, the analysis performed using a randomeffects model. When heterogeneity was not significant among the results of the study, the Fix 
effects model was used to pool analysis and verses. Publication bias was measured with Begg's adjusted rank correlation test and graphically depicted by funnel plot.

\section{RESULTS}

Upon completion of the search, 22 studies with a 10234 sample size were included into the metaanalysis (Flowchart 1). The prevalence of Leptospirosis as well as gender, age and occupation parameters was extracted. Our analysis showed that the prevalence of Leptospirosis in Iran is $39 \%$ (95\% CI 29-49) (fig 1). It was interesting that while the highest prevalence was obtained from Gilan province (70\%; 95\% CI 67-73) (with sample size of 995 people); the lowest prevalence was found in Golestan province (10\%; 95\% CI 9-12) (with sample size of 1025 people) (Table 1). For detailed information about the prevalence of Leptospirosis in Iran, refer to Table 1. According to the analysis based on city and/ or province, the highest prevalence rate 44\% with a sample size of 1505 people was seen in Mazandaran province (95\% CI 27-61) and the lowest prevalence rate of $34 \%$ with a sample size of 1048 people was seen in Golestan province ( 95\% CI 15-83) (fig 2 ). Generally, analysis based on different geographical regions in Iran demonstrated that the prevalence of Leptospirosis in the northern part of Iran was 41\% (95\% CI 29-53) with a 9191 sample size. The prevalence rate in central Iran was $49 \%$ (95\% CI 44-53) with sample size of 480 (fig 3).

Prevalence of Leptospirosis in males was 69\% (95\% CI 74-56) and in females was 35\% (95\% CI 44-26). When occupation was subjected for evaluation, the highest prevalence -as expected- was seen in farmers (65\%) (95\% CI 74-57) (Fig. 4) and the lowest prevalence belonged to students (4\%) (95\% CI 7-1). The analysis based on age groups demonstrated a $40 \%$ (95\% CI 34-46) prevalence among the age group of 40-60 years, $16 \%$ (95\% CI 10-22) among the 
age group $\geq 60$ years and $9 \%$ (95\% CI 5-13) among the age group of 0-20 years. The sensitivity for MAT was estimated to be 39\% (95\% CI 30-48), while for ELISA was 49\% (95\% CI 27-71) and for IFA was 54\% (95\% CI 8-100) (Fig. 5).

Furthermore, meta-regression analysis demonstrated that the prevalence of Leptospirosis has slightly decreased from 1995 to 2014 and was statistically significant ( $\mathrm{p}=0124$ ) (Fig. 6), however there was no significant correlation between prevalence and sample size ( $\mathrm{p}=0.58)$ (Fig. 7). Also funnel plot showed there was no publication bias (Fig. 8)

\section{DISCUSSION}

Our meta-analysis demonstrated that the prevelance of Leptospirosis in Iran according to age, gender, and occupation parameters is $39 \%$. Most outbreaks of Leptospirosis, which indicates in the majority of farmers, are occupational and the disease is more prevalent in wet climates. Alayian and colleagues observed a significant correlation between Leptospirosis and occupation and suggested a special control program for susceptible individuals (9). The majority of reports indicated that Leptospirosis is more prevalent among farmers. In agreement with Faraji and colleagues, our results showed that $65 \%$ of all studied patients had agricultural occupation (10). Taleei and colleagues showed that $49 \%$ of rice farmers were seropositive while only $15 \%$ of ranchers were seropositive. In agreement with this meta-analysis results, they indicate a high prevalence of Leptospirosis among farmers and so it seems that this group is at a high risk of developing Leptospirosis (11). 
Perret and colleagues reported $72 \%$ of farmers have Leptospirosis. Slack et al considered type of occupation and exposure as main determinants in development of this disease $(12,13)$. Most of Leptospirosis outbreaks occurred in tropical regions including Asia, Pacific, Latin America, and Caribbean especially after floods and recently it was seen in Nicaragua and Philippines (14).

Leptospirosis was more prevalent among males than females, as showed by previous studies (1518). Reports from European countries also showed that the risk ratio of developing Leptospirosis in males is four times more likely than females (19). The results of the current study also showed that Leptospirosis was more prevalent among patients aged 20-60 years old. This is confirmed by the other studies in Iran $(11,17,20-21)$. In agreement to our results, Fero and colleagues also showed Leptospirosis was observed mostly in patients over 50 years old in Colombia (22).

Despite the deficiency of studies about diagnostic methods for leptospirosis in Iran, it seems that rapid leptospirosis antibody based tests such as ELISA are used frequently $(23,6,11$, 15, 23-28). Similar to the above studies, our results showed higher prevalence of Leptospirosis by ELISA (49\%) than MAT (39\%) in Iran. The gold standards for diagnosis of Leptospirosis are culture and the microscopic agglutination test (MAT) which needs specific equipments and highly trained staff (29). Culture is not suitable for the diagnosis of the slow-growing Leptospira spp, and MAT which detects anti-Leptospira antibodies only in the second week of the disease, cannot detect early stages of the disease $(29,30)$. Some studies reported that other techniques can detect anti-leptospira antibody earlier in the course of the disease than MAT. Although antileptospira IgM antibodies are not detectable before 4-5 days after the onset of the disease, but appear earlier than IgG and agglutinating antibodies (29, 31). So, although ELISA cannot identify Leptospira serovars but could be applied as a routine diagnostic and primary screening 
test especially in endemic regions. According to some researches, it seems that semi-quantitative ELISA is more valuable in Iran because of its high sensitivity and specificity (24-27).

Finally, it could be concluded that Leptospirosis occurred highly in tropical and temperate climates, especially in hot and humid areas of northern Iran such as Gillan and Mazandaran provinces. Although meta-regression analysis shows that during 1997 and 2012 the prevalence of Leptospirosis has decreased slightly in Iran and was statistically significant, but due to lack of Leptospirosis specific clinical symptoms and differences in sensitivity and specificity of diagnostic tests, we cannot conclusively explain this decrease.

Although using rapid diagnostic tests such as ELISA and PCR and conventional methods such as culture and MAT can be very important in diagnosis and starting the treatment, but to greatly reduce Leptospirosis occurrence, we should note that prevention of Leptospirosis must be considered as a basic principle. Leptospirosis is a zoonotic and human disease only. Avoidance of human exposure to infected animals or their tissue, urine and blood, wearing protective clothing such as gloves and boots especially for people at high risk such as veterinarians, farm workers and sewer workers, not swimming or wading in water contaminated with the urine of infected animals, can be very helpful in controlling Leptospirosis in Iran. 


\section{REFERENCES}

1. Zavitsanou A, Babatsikou F. Leptospirosis: epidemiology and preventive measures. Health. Sci. J. 2008; 2:75-82.

2. Adesiyun A, Hull-jackson C, Mootoo N, Halsall S, Bennett R, Clarke N. Sero-epidemiology of Canine Leptospirosis in Trinidad: Serovars, Implications for Vaccination and Public Health. J Vet Med. 2006; 53: 91-9.

3. Lilenbaum W, Morais ZM, Gonçales AP, Souza GO, Richtzenhain L, Vasconcellos SA. First isolation of leptospires from dairy goats in Brazil. Brazilian J Microbiol. 2007; 38: 507-10.

4. Izurieta R, Galwankar S, Clem A. Leptospirosis: The "mysterious" mimic. J Emerg Trauma Shock. 2008; 1: 21-33.

5. Sharma, J, Suryavanshi M. Thrombocytopenia in leptospirosis and role of platelet transfusion. Asian J Transfus Sci. 2007; 1: 52-5.

6. Victoriano AF, Smythe LD, Gloriani-Barzaga N, Cavinta LL, Kasai T, Limpakarnjanarat K. Leptospirosis in the Asia Pacific region. BMC Infect. 4: 147.

7. Peces, R. 2003: Acute renal failure in severe leptospirosis. Nephrol Dialy Transplant. 2009; 18: 1235-6.

8. Araujo ER, Seguro AC, Spichler A, Magaldi AG, Volpini RA, De Brito T. Acute kidney injury in human leptospirosis: an immunohistochemical study with pathophysiological correlation. Virchows Archiv. 2010; 456: 367-75.

9. Aliyan S, Babamahmoudi F, Najafi V, Qasemian R, Teymouri S, Shahbaznezhad L. Clinical and Para clinical findings of leptospirosis in Mazandaran, June-September 2004. J Mazandaran Uni Med Sci. 2006; 16:78-85. 
10. Faragi H, Assmar M, Ebrahimipour GH, Hashemi Chelavi S, Esfandiari B, Ziapour S. The survey of seroprevalence of leptospirosis in mazandaran province of iran using microscopic agglutination test and indirect immunofluorescence assay. iranian journal of biology. Iran J Biol. 2010; 22: 608-618.

11. Taleei G, Sheikhian A, Mousavi SZ. Seroprevalance of leptospirosis in Farmers of Vaysian and Ranchers of Azna in autmn 2006. Yafte. 2007; 9: 3-9.

12. Perret PC, Abarca VK, Dabanch PJ, Solari GV. Risk factors and frequency of positive antibodies for leptospirosis in a sub urban population near Santiago. Rev Med Chil. 2005; 133: 426-431.

13. Slack A. Leptospirosis. Aust Fam Physician. 2010; 39: 495-498.

14. Taylor AJ, Paris DH, Newton PN. A Systematic Review of the Mortality from Untreated Leptospirosis. PLoS Negl Trop Dis. 2015; 9: e0003866.

15. Honarmand H, Eshraghy S, Khoramizadeh MR, Mansour GF, Hartskerrk R. Identifying serogroup and servers of acute human leptospirosis in gillan province by mat method. J Gilan Univ Med Sci. 2006; 15: 8-14.

16. Honarmand HR, Eshraghi SS. Comparison of two ELISA methods for the laboratory diagnosis of acute leptospirosis. IJMS. 2010; 35: 116-121.

17. Rahimi F, Vand Yousefi J, Moradi Bidhendi S, Bouzari M. Leptospirosis in the rural areas of Guilan province (2004-2005). J Kermanshah Uni Med Sci. 2007; 11: 197-205.

18. Ghanaei FM, Fallah MS, Jafarshad R, Joukar F, Honarmand H, Heidarzadeh A, and Khoshsorur MEpidemiologic assessment of Leptospira serotypes in Caspian littoral. Iran J Clin Infect Dis. 2008; 3: 133-136. 
19. Dupouey J, Faucher B, Edouard S, Richet H, Kodjo A, Drancourt M, Davoust B. 2014: Human leptospirosis: An emerging risk in Europe? Compar Immunol Microbiol Infect Dis. $2014 ; \mathbf{3 7 : 7 7 - 8 3 . ~}$

20. Majd NS, Darian EK, Khaki P, Bidhendi SM, Yahaghi E, Mirnejad P. Epidemiological patterns of Leptospira spp. among slaughterhouse workers in Zanjan-Iran. Asian Pac J Trop Dis. 2012; 12: S550-S2.

21. Esfandiari B, Youssefi MR, Asmar M. Seroprevalence of leptospirosis in north of Iran during 2010. World App Sci J. 2011; 14:1296-8.

22. Ferro B, Rodríguez A. Seroprevalence of Leptospira infection in habitants of peripheral neighborhoods in Cali, Colombia. Biomed. 2006; 26: 250-257.

23. Javid N, Dadgar T, Khodabakhshi B, Bazouri M, Sedaghat M, Bakhshandeh-Nosrat S, Rahimi S, Ghaemi E. Seroepidemiology of anti-leptospira antibody in Golestan province, north of Iran. Int. J Mol Clin Microbiol. 2012; 2: 124-127.

24. Honarmand $\mathrm{H}$, Khayat L. Isolation of pathogenic leptospires from blood samples of patients by PCR-RFLP method. J Gorgan Univ Med Sci. 2010; 12: 70-9.

25. Honarmand H, Resaei H, Rezvani M, Hosseini S, Rahbar M. Ten years incidence of leptospirosis in guilan (1999 to 2008). Iran. J Infect Dis Trop Med. 2010; 47: 47-53.

26. Honarmand H, Nezafat Tabalvandi M, Heydarzadeh A, Soltani B, Mirzajani E. Evaluation an in-house IgM-ELISA for the diagnosis of human leptospirosis. Koomesh. 2008; 9: 309-314.

27. Honarmand H, Eshraghi S, Khorramizadeh M, Hartskeerl R, Ghanaei F, Abdolahpour G. Distribution of Human Leptospirosis in Guilan Province, Northern Iran. Iran J Public Health. 2007; 36, 68-72. 
28. Taghavi SH, Nabavi M, Amini S R R. Serological study for measuring rate of leptospirosis in patients who encountered "shaltook fever". Acta Med Iran. 2006; 44: $131-4$.

29. Picardeau M, Bertherat E, Jancloes M, Skouloudis AN, Durski K, Hartskeerl RA. Rapid tests for diagnosis of leptospirosis: current tools and emerging technologies. Diagn Microbiol Infect Dis. 2014; 78: 1-8.

30. Bharti AR, Nally NE, Ricaldi JN, Matthias MA, Diaz MM, Lovett MA, Levett PN, Gilman RH, Willig MR, Gotuzzo E, Vinetz JM. Leptospirosis: a zoonotic disease of global importance. Lancet Infect Dis. 2003; 3: 757-71.

31. Aviat F, Rochereau-Roulet S, Branger C, Estavoyer JM, Chatrenet B, Orsonneau JL, Thorin C, Andre-Fontaine G. Synthetic peptide issued from Hap1/LipL32 for new early serodiagnosis of human leptospirosis. Comp. Immunol. Microbiol. Infect. Dis. 2010; 33: $375-87$.

32. Ebrahimi A, Alijani L. Serological Survey of Human Leptospirosis in tribal Areas of West Central Iran [hplimg]. IJMS. 2003; 28: 93-95.

33. Babamahmoodi F, Mojaveri MS, Babamahmoodi A. Seroepidem ology of leptospirosis in workers of high risk occupation in Mazandaran province-Iran 2007-2008. J Mazandaran Uni Med Sci. 2009; 19: 10-5.

34. Ziapour S, Esfandiari B, Abdollahpour G, Assmar M, Youssefi M, Amirbozorgi G. The Survey of leptospirosis in Mazandaran province, north of Iran by Microscopic agglutination test, 2006-2007. Int J Infect Dis. 2010; 14: e206. 
35. Golsha R, Khodabakhshi B, Rahnama A. Leptospirosis in Golestan province in Iran (Reports of twelve cases). J Gorgan Uni Med Sci. 2007; 9: 76-80.

36. Haji Hajikolaei MR, Ghorbanpour M, Komeylian MM, Abollapour GR. A study of leptospiral infection among the people of ahwaz with direct and indirect contact with animal. Iran Vet J. 2008; 4: 14-23.

37. Mansour-Ghanaei F, Sarshad A, Fallah MS, Pourhabibi A, Pourhabibi K, YousefiMashhoor M. Leptospirosis in Guilan, a northern province of Iran: assessment of the clinical presentation of 74 cases. Med Sci Monit. 2005; 11: CR219-CR23.

38. Sakhaee E. Detection of leptospiral antibodies by microscopic agglutination test in northeast of Iran. Asian Pac J Trop Biomed. 2011; 1: 227-9. 
Table1: Characteristics of papers included in the meta-analysis

\begin{tabular}{|c|c|c|c|c|c|}
\hline Reference & year & Province & Total sample & $\begin{array}{l}\text { Positive } \\
\text { sample }(\%)\end{array}$ & method \\
\hline Majd et al. (2012) & 2012 & Zanjan & 98 & 35 & MAT \\
\hline Javid et al. (2012) & 2008 & Golestan & 1028 & 10 & ELIASA \\
\hline Esfandiari et al. (2011) & 2010 & Mazandaran & 688 & $27 / 5$ & IFA \\
\hline Honarmand \& Eshraghi (2010) & 2010 & Guilan & 282 & 25 & ELIASA \\
\hline Ebrahimi \& Alijani (2003) (32) & 2002 & Chaharmahal & 400 & $48 / 5$ & MAT \\
\hline $\begin{array}{l}\text { Babamahmoodi et al. (2009) } \\
\text { (33) }\end{array}$ & 2009 & Mazandaran & 500 & 14 & IFA \\
\hline Honarmand et al. (2006) & 2003 & Guilan & 282 & 24 & ELIASA \\
\hline Ziapour et al. (2010) (34) & 2006 & Mazandaran & 127 & 53 & MAT \\
\hline Golsha et al. (2007) (35) & 2004 & Golestan & 20 & 60 & MAT \\
\hline Alian et al. (2006) & 2004 & Mazandaran & 63 & 69 & MAT \\
\hline Faraji et al. (2010) & 2006 & Mazandaran & 127 & 58 & MAT \\
\hline Rahimi et al. (2007) & 2004 & Guilan & 552 & 21 & MAT \\
\hline Hajikolaei et al.(2008) (36) & 2004 & Khuzestan & 180 & 18 & MAT \\
\hline Taleie et al. (2007) & 2005 & Lorestan & 80 & 49 & ELIASA \\
\hline Honarmand et al.(2010) & 1999 & Guilan & 3431 & 60 & ELIASA \\
\hline Ghanaei et al. (2008) (37) & 2004 & Guilan & 465 & 38 & MAT \\
\hline Taghavi et al. (2006) & 2002 & Guilan & 87 & 30 & ELIASA \\
\hline Sakhaee et al (2011) (38) & 2009 & Kerman & 285 & 16 & MAT \\
\hline Mansour-Ghanaei et al.(2005) & 1999 & Guilan & 237 & 31 & IFA \\
\hline Honarmand et al.(2008) & 2006 & Guilan & 200 & 49 & ELIASA \\
\hline Eshraghi et al.(2007) & 2003 & Guilan & 995 & 70 & ELIASA \\
\hline Honarmand et al.(2010) & 2008 & Guilan & 107 & 60 & PCR \\
\hline
\end{tabular}




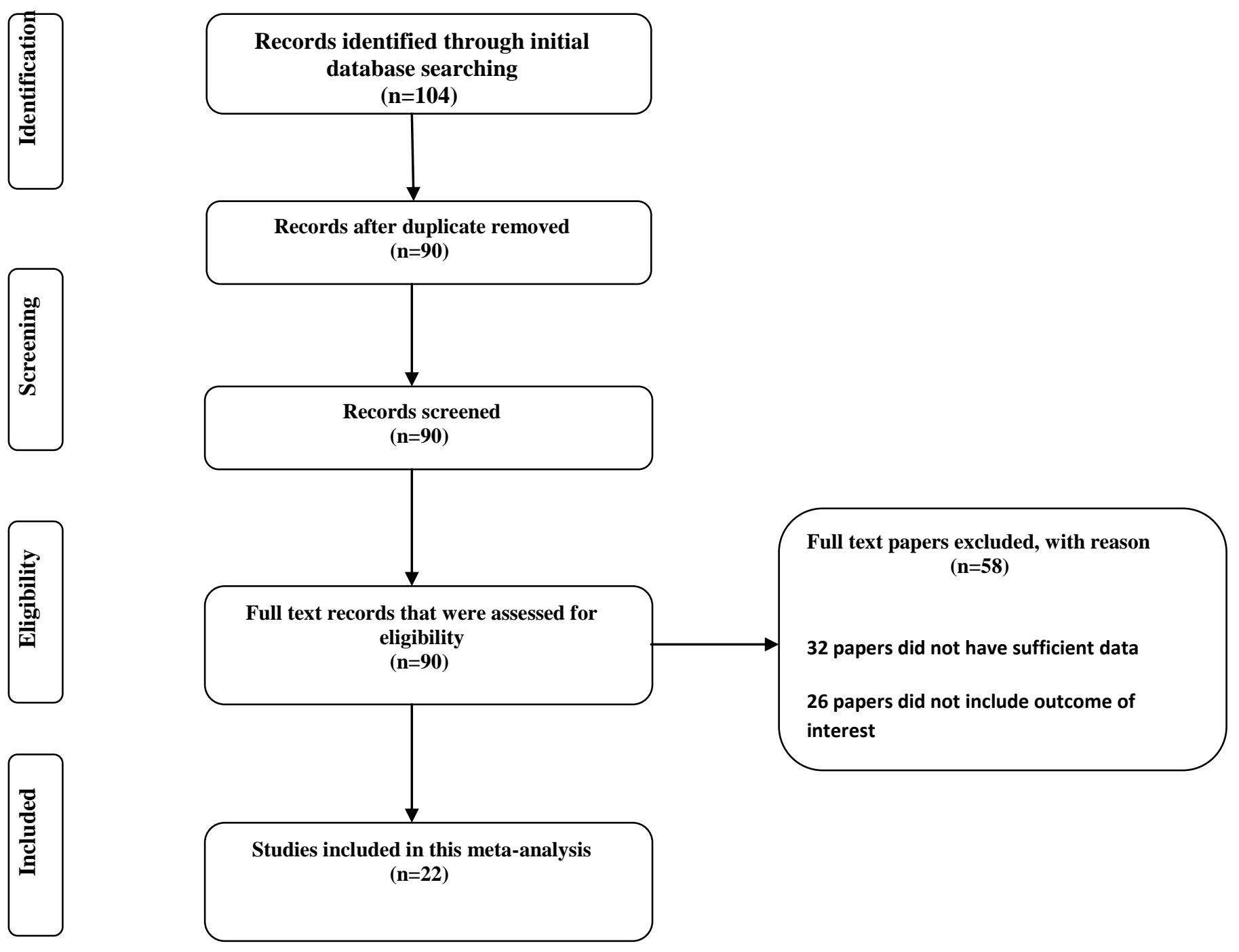

Flowchart 1. Studies selection for meta-analysis 


\begin{tabular}{l|rr} 
Study \\
ID
\end{tabular}

Figure 1: The prevalence of Leptospirosis in each of the studies. The segments show a $95 \%$ prevalence of Leptospirosis. Diamond mark indicates the estimated overall prevalence rate. 


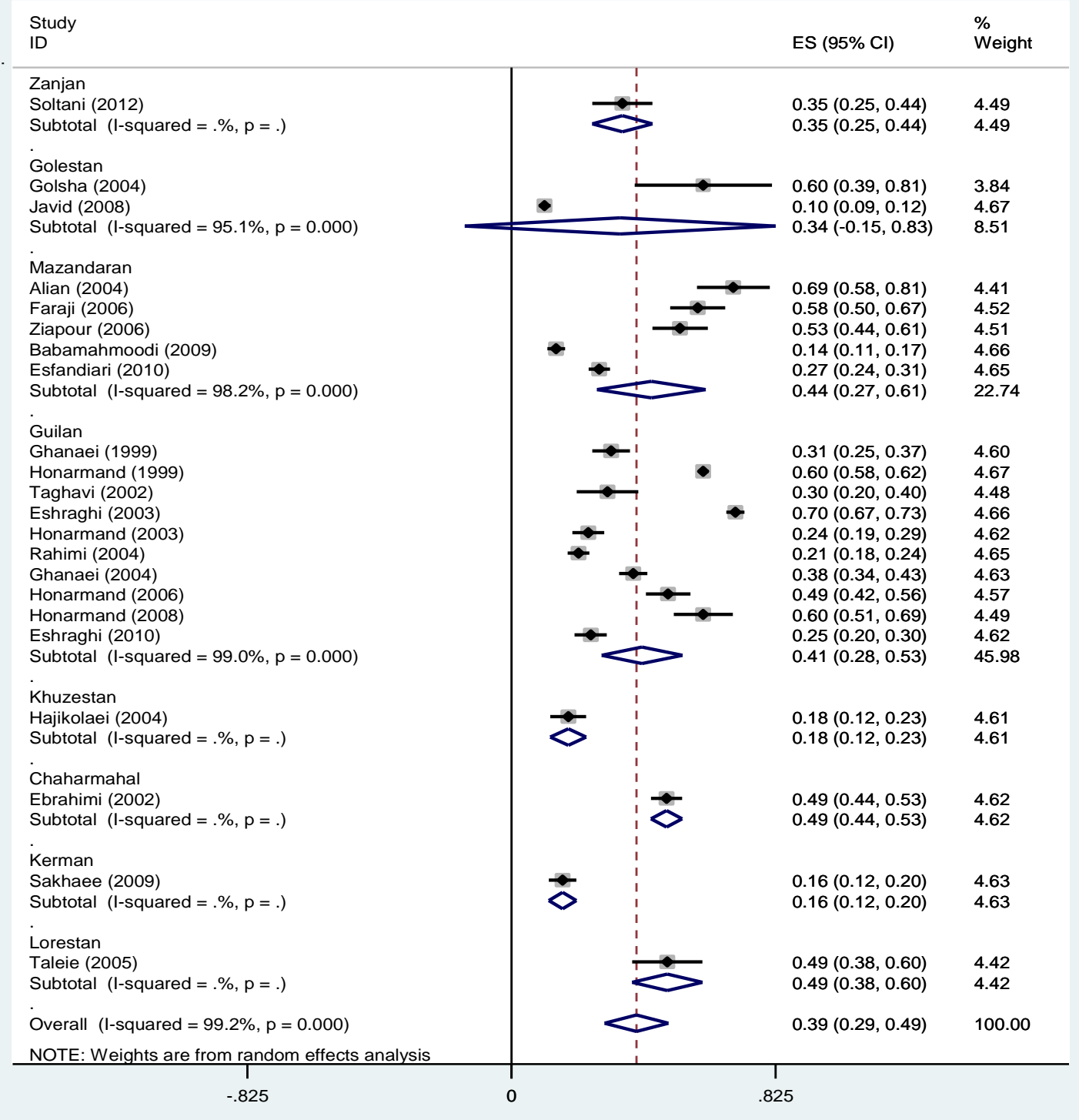

Fig 2: The prevalence of Leptospirosis in different cities. The segments show a $95 \%$ prevalence of Leptospirosis. Diamond mark indicates the estimated overall prevalence rate. 


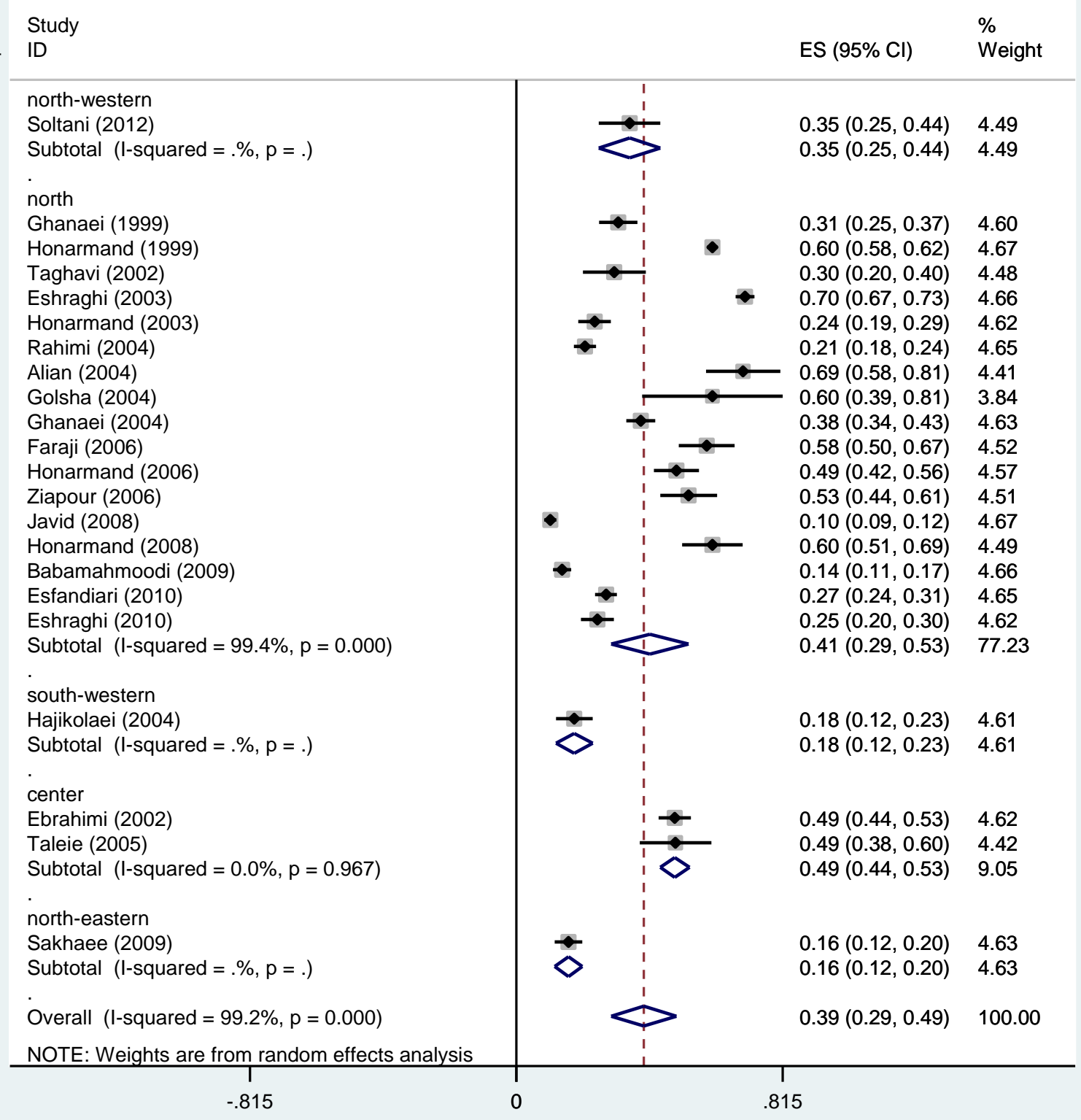

Fig 3: The prevalence of Leptospirosis in different geographical regions of Iran. The segments show a 95\% prevalence of Leptospirosis. Diamond mark indicates the estimated overall prevalence rate. 


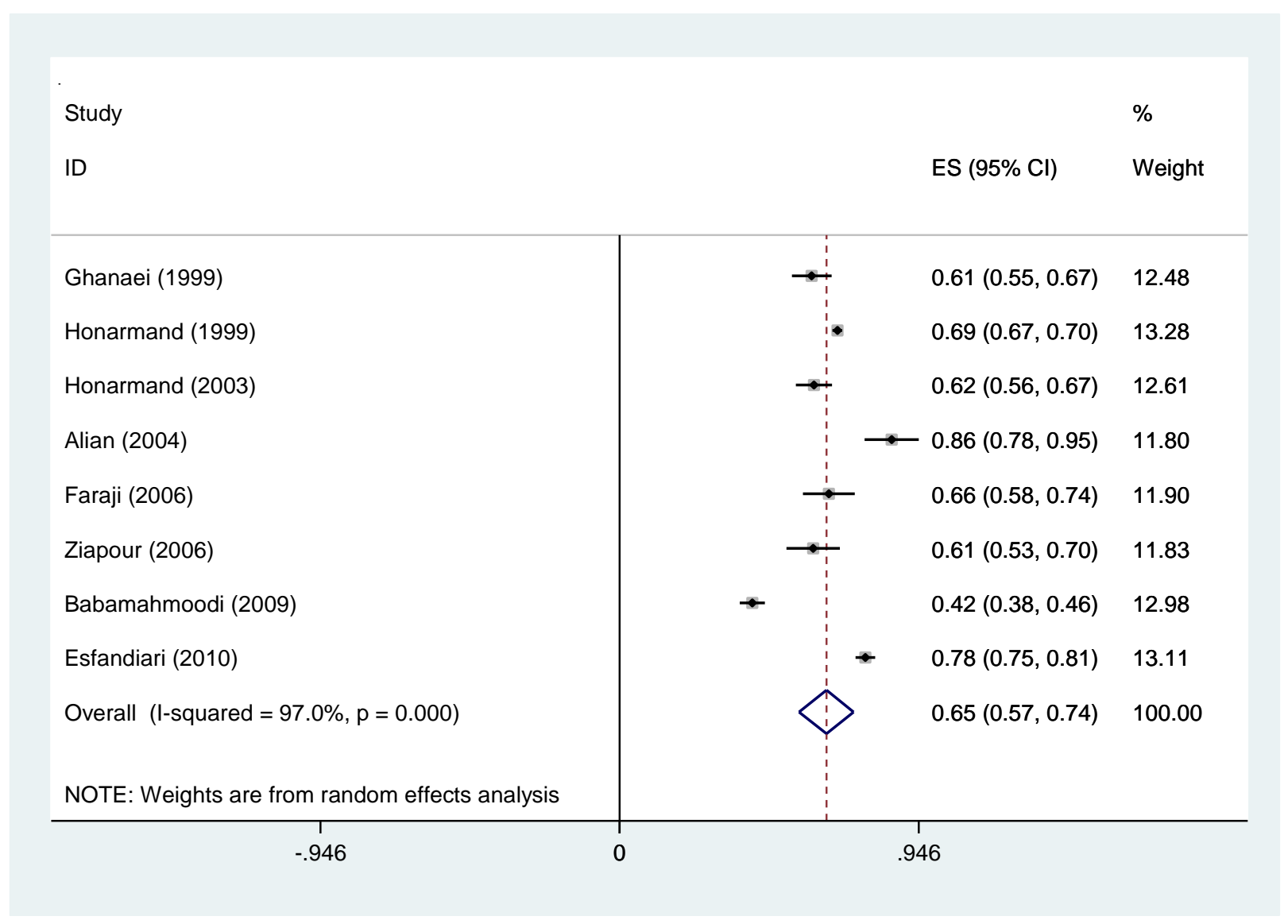

Fig 4: The prevalence of Leptospirosis among farmers. The segments show a 95\% prevalence of Leptospirosis. Diamond mark indicates the estimated overall prevalence rate 


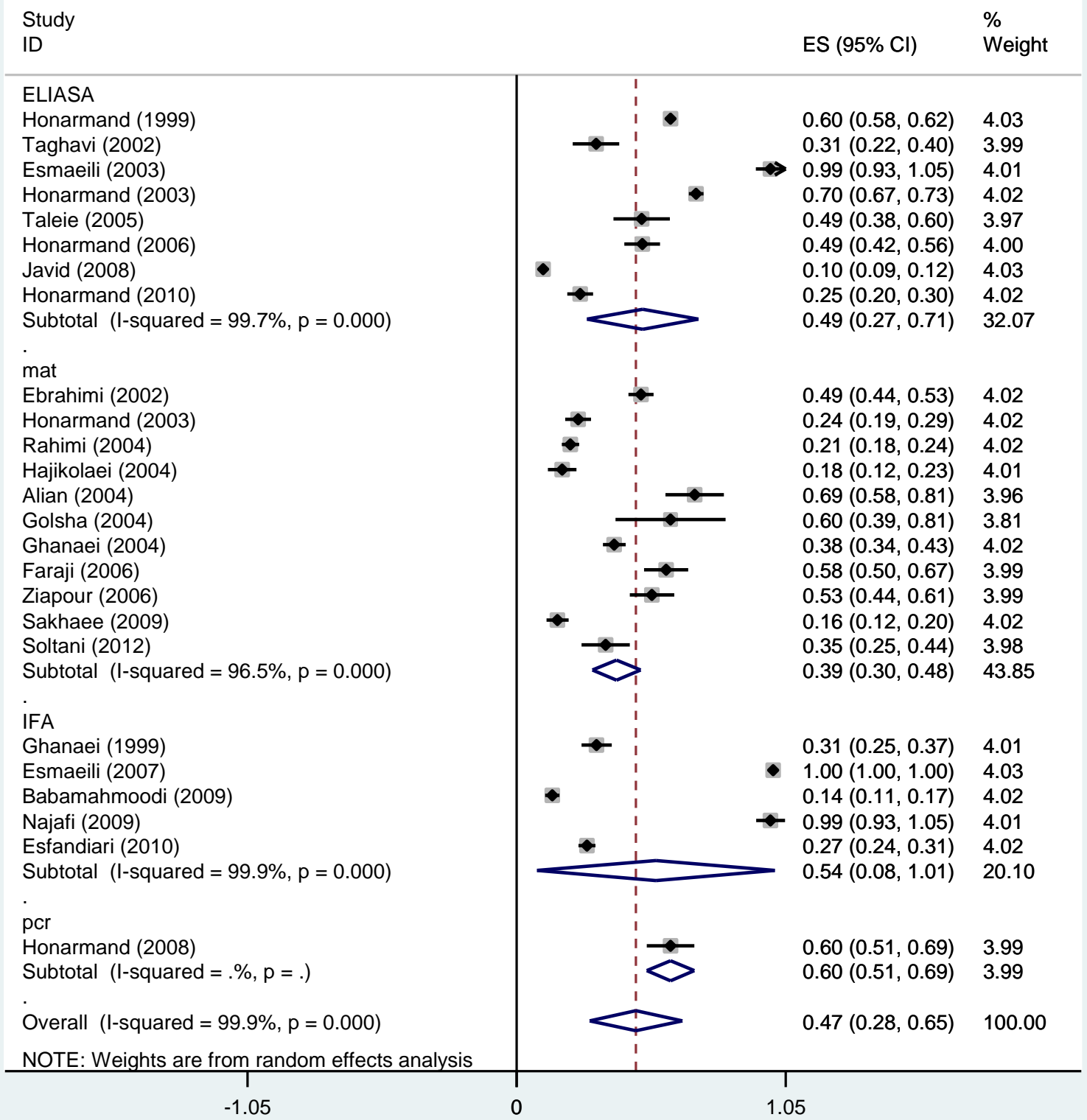

Fig 5: Prevalence of Leptospirosis in Iran, according to different diagnostic methods 


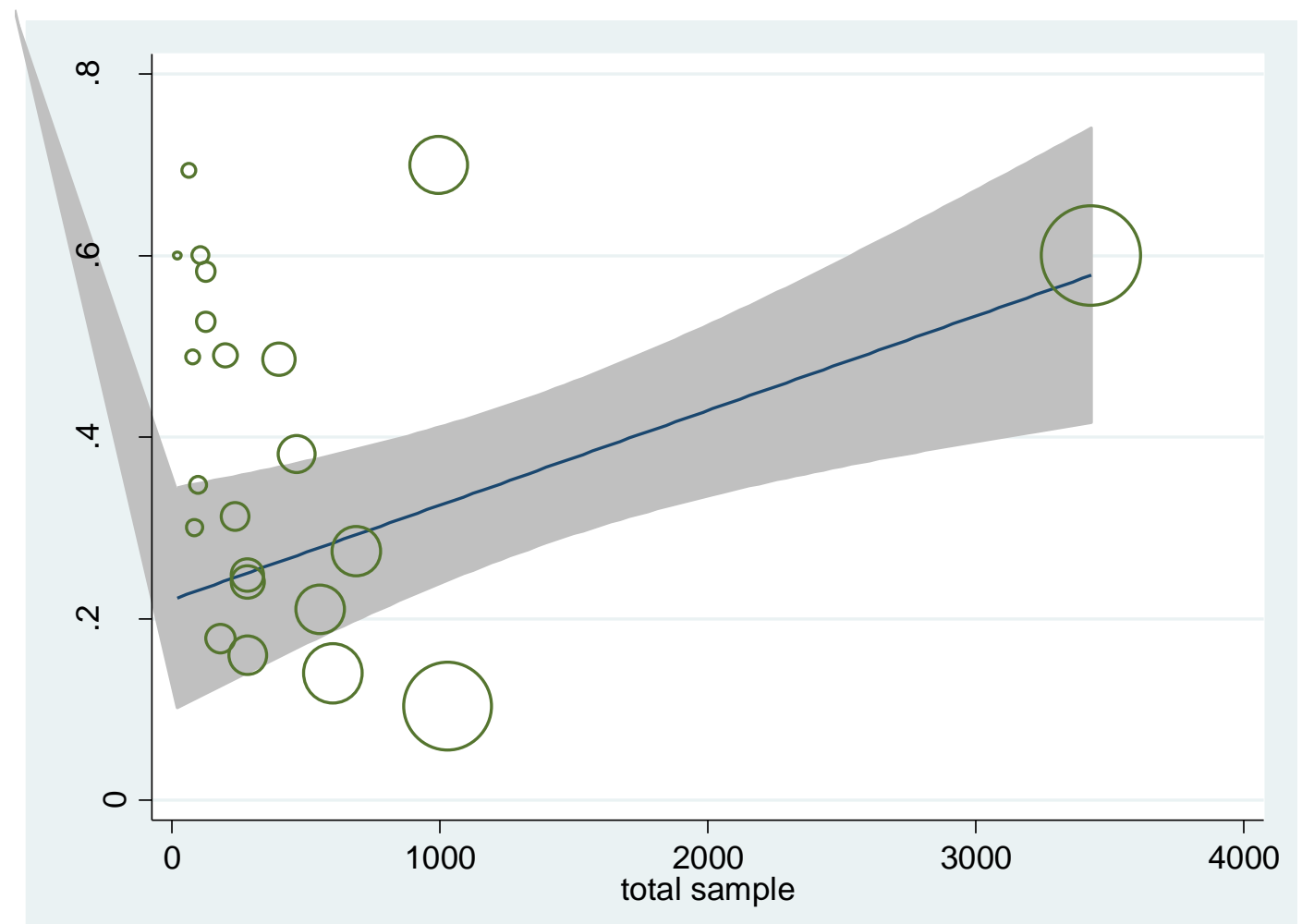

Fig 6: Meta-regression. The relationship between the prevalence of the disease and the sample size shows that the prevalence also increased with increasing sample size. But there was no statistically significant relationship. $\mathrm{P}=0.586$. (Size of the circle indicates the sample size and larger circles represent more samples). 
Khayat et al

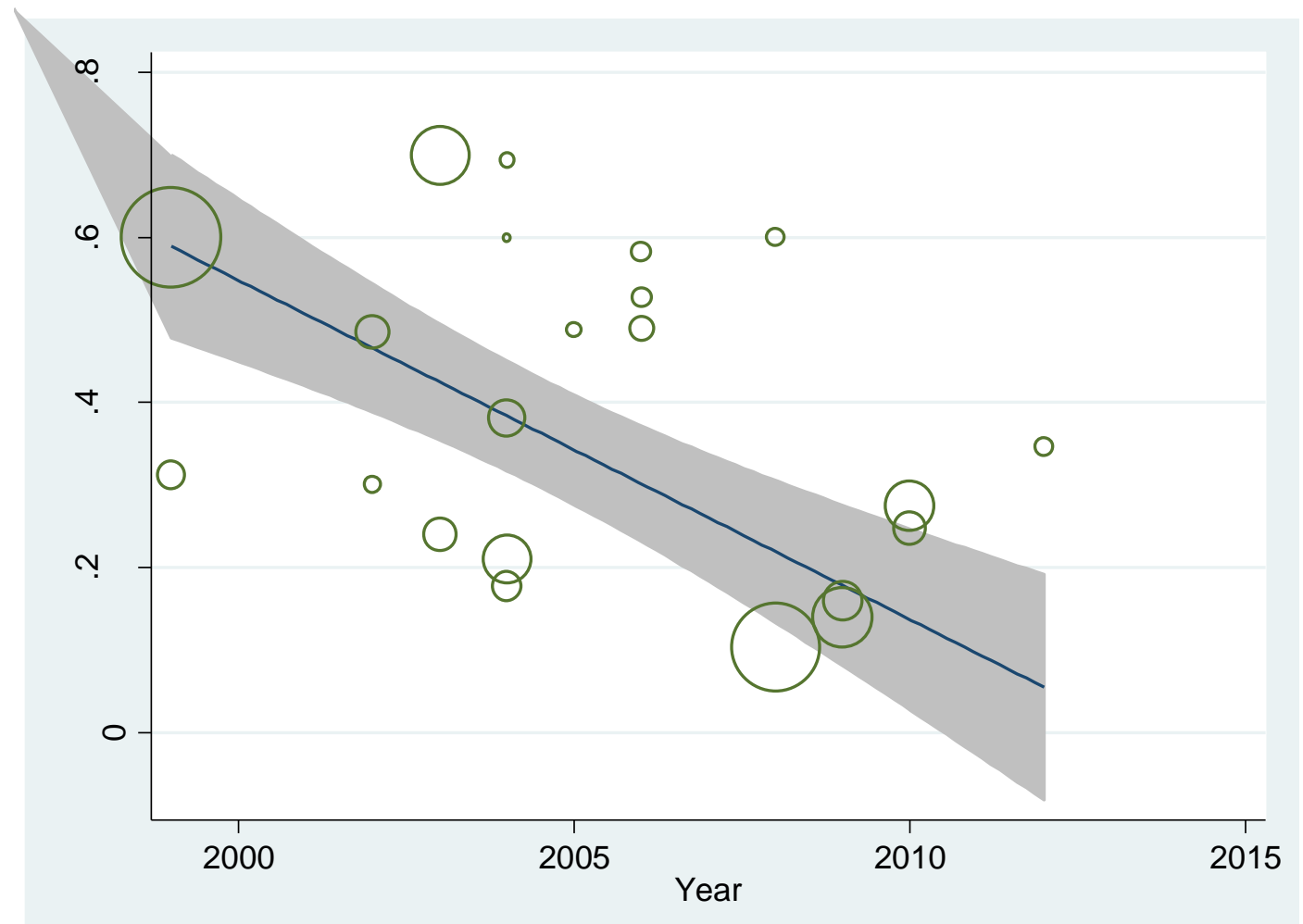

Fig 7: Meta-regression. The relationship between the prevalence of the disease and the time parameter (year) shows that during 1997 and 2012 the prevalence of the disease has decreased slightly and was statistically significant. $\mathrm{P}=0.124$ (size of the circle indicates the number of samples and more samples are indicative of a larger circle.) 


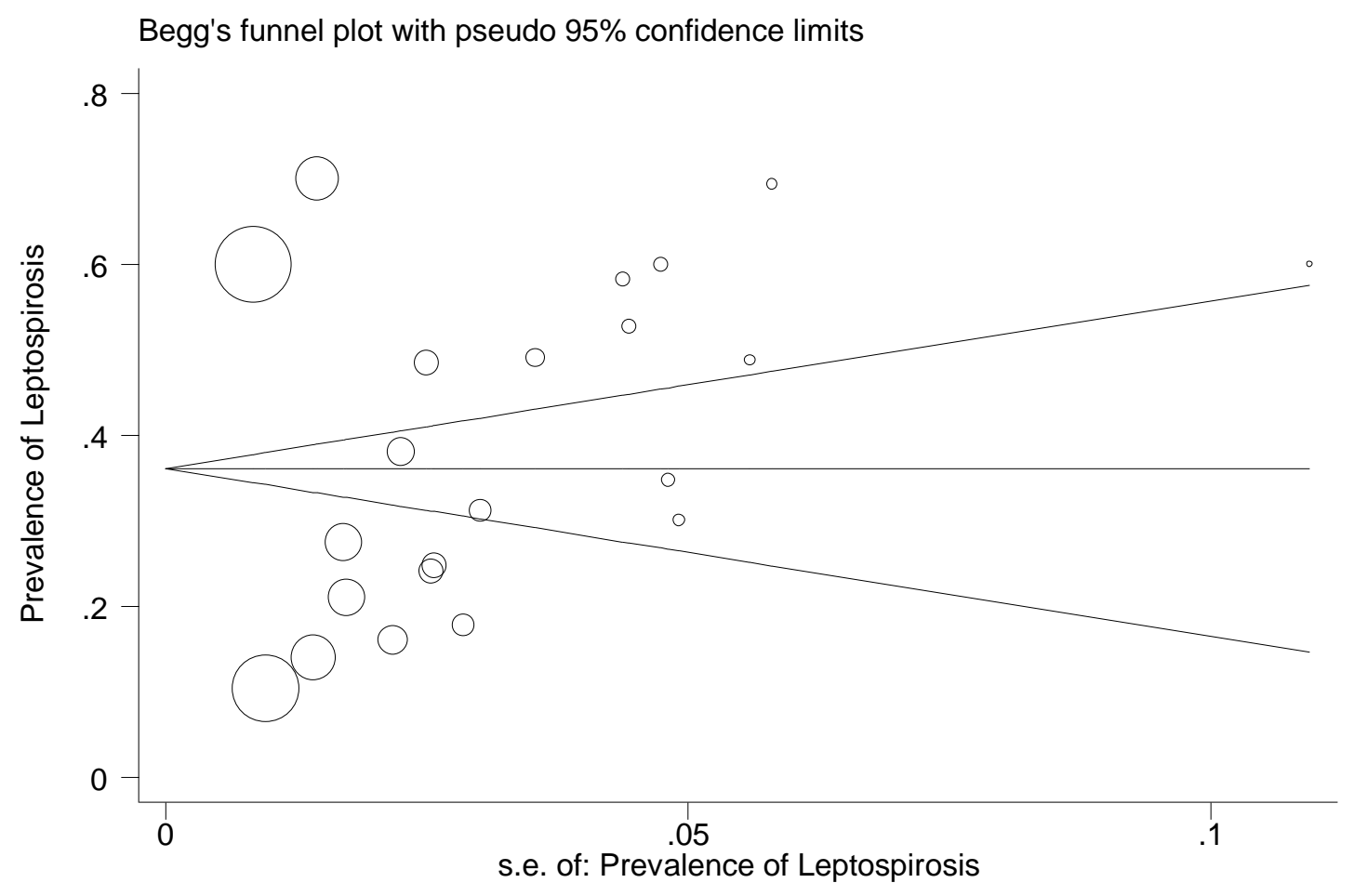

Fig 8: Funnel plot for assessing publication bias 